\title{
Citizen Scientists Engagement in Air Quality Measurements
}

\section{Prof. Anthony Butterfield, University of Utah}

Anthony Butterfield is an Assistant Professor (Lecturing) in the Chemical Engineering Department of the University of Utah. He received his B. S. and Ph. D. from the University of Utah and a M. S. from the University of California, San Diego. His teaching responsibilities include the senior unit operations laboratory and freshman design laboratory. His research interests focus on undergraduate education, targeted drug delivery, photobioreactor design, and instrumentation.

Katrina My Quyen Le, AMES High School 


\title{
Citizen Scientists Engagement in Air Quality Measurements
}

\begin{abstract}
:
Citizen scientist efforts, wherein members of the public who are not professional scientists participate in active research, have been shown to effectively engage the public in STEM fields and result in valuable data, essential to answering pressing research questions. However, most citizen scientist efforts have been centered in colleges of science, and a limited number have crossed into research areas important to chemical engineering fields. In this work we report on the results of a project to recruit high school and middle school students across Utah's Salt Lake Valley as citizen scientists and potential engineering students who work in partnership with chemical engineering researchers in an effort to create a distributed online network of air quality sensors.

Middle and high school students were trained by undergraduate mentors to monitor and maintain their own outdoor air quality sensor with the help of teaching materials that were co-developed with Breathe Utah, a local community group concerned with air quality. With the help of these tailored teaching modules, students learned about the science behind air quality research and the difficulties common to physical measurements to better prepare them to analyze their data. Once trained, students are expected to become semi-independent researchers in charge of monitoring and maintaining their piece of a larger air quality map.

We describe in this work the hurdles inherent in citizen science engagement within a chemical engineering research program and the means to address them. We describe successful means of engaging classrooms, training citizen scientists, obtaining faculty buy-in within the confines of state curricular demands, and addressing school administration concerns.

With this model, we have directly engaged over 1,000 high school and over 3,000 middle school students. The project has resulted in a growing network of citizen-maintained sensors that contributes to a real-time air quality map. Student scientists may also use the sensors to participate in active research or conduct science fair projects. Student response to this citizen scientist project, where it may be measured, has been enthusiastic and almost wholly positive.
\end{abstract}

\section{Introduction:}

Citizen scientist (CS) projects generally engage a large number of non-professional scientists in conducting novel research. Most often, the work conducted depends greatly on recruiting and training many amateur researchers to contribute to scientific tasks which would not be manageable for a typical university research group. Such work may also be referred to as crowdsourced science, or community science.

While engaging the general public in scientific research is not a new concept, the ability to connect and coordinate vast numbers of volunteers over the internet has facilitated a recent boom in CS work [1]. Several projects have been remarkably successful, capturing national attention and engaging hundreds of thousands of citizen researchers. The Zooniverse project (https://www.zooniverse.org/), for example, recruits the public to use their home computers to view and categorize galaxy types [2]. Another prominent example is Foldit (https://fold.it/), an 
online game wherein players compete to predict protein folding. Such work has led to significant scientific contributions. Zooniverse has, to date, resulted in over 50 peer-reviewed publications, and the top players/citizen researchers of Foldit have proven themselves to be even more accurate at predicting protein folding than state-of-the-art algorithms [3].

Specifically within the area of environmental science, a recent report from the Environmental Protection Agency "identifies citizen science not only as an invaluable opportunity for the Agency to strengthen public support for EPA's mission but also as the best approach for the Agency to connect with the public" [4]. While CS efforts have the attention of the scientific community and governmental funding sources, they have generally not found use in chemical engineering departments or engineering departments in general. Primarily, CS projects have been concentrated within colleges of science, with the top areas of research focus being biology and ecology [5]. Furthermore, most of the existing and prominent CS projects rely on the public to provide their own sensors and visualization devices in the form of mobile devices and personal computers.

In this work we describe effective engagement methods and results from a CS chemical engineering research project to quantify, track, and determine the sources of poor air quality (AQ) in the Salt Lake Valley by providing laypersons with low-cost, internet-enabled AQ sensors, called AirU sensors. This project focusses primarily on the unique hurdles of partnering with middle and high school CS participants, particularly where the involvement of school district governance is required to both generate useful research data and facilitate STEM recruitment.

\section{Broad CS Project Model}

Figure 1 shows a depiction of the broad pieces of this CS model. This CS project is a part of a larger research program called the AQ\&U Project, which is described in detail online, both at https://airu.coe.utah.edu/ and http://www.aqandu.org/. In summary, the aim of the AQ\&U

Project is to establish a valley-wide network of low-cost, air quality sensors. This network is to be used to answer research questions about: the sources and distribution of poor air quality; the

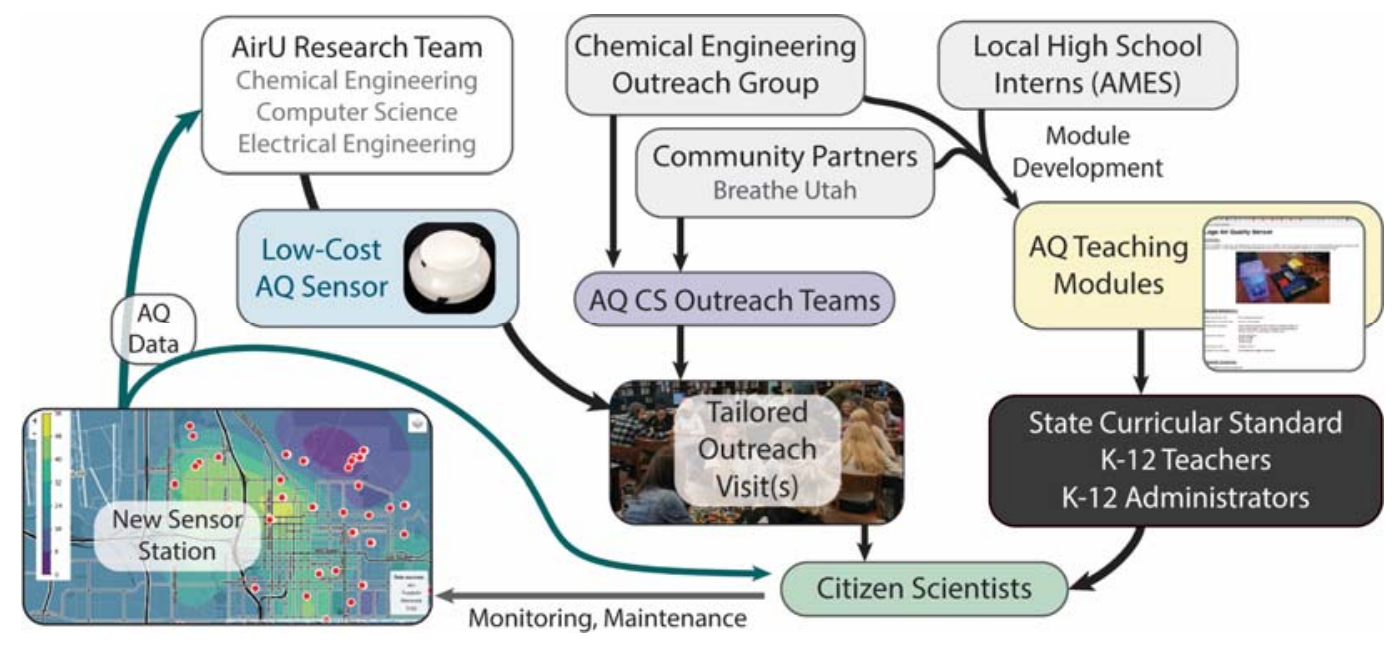

Figure 1: AirU CS Project Model 
performance of the low-cost sensors; strategies to integrate information from various imperfect measurements; and means of addressing the poor air quality that often settles in the populated valleys around the globe.

In addition to $\mathrm{Wi}-\mathrm{Fi}$ access and power, each sensor requires monitoring, maintenance, and an understanding of possible neighborhood sources of poor air quality in order to best interpret observations. As such, distributed citizen involvement becomes an ideal means to execute such research. Citizens, as groups or as individuals, are first instructed on the workings and maintenance of the AirU sensors (which are assembled in-house). A sensor is then placed in a sheltered area outside the citizens' home, school, or workplace. Subsequently, the citizens monitor their data for proper performance, reporting on relevant events in their area. Approximately 150 sensors are part of this larger project, 50 of which are meant to be used in partnership with local middle and high schools in this CS project focused on engaging potential engineering students. These sensors connect to the citizen scientist's Wi-Fi network and feed data to servers at the University of Utah. This data is used for research purposes and to create an online real-time air quality map as seen here: (http://www.aqandu.org/).

In the K-12 outreach portion of this work we relied on our established chemical engineering outreach model, described elsewhere [6], in which we use both paid and volunteer undergraduate students to develop outreach teaching modules and conduct events at regional schools, summer camps, and community events. This program began as an NSF-funded project over eight years prior and has since become a self-sustained department organization, reaching well over 1,000 students each year. To justify the expense of such a substantial outreach program, the group has transitioned to partnering with our research programs, helping to facilitate broader impacts and communication of our department's research.

The Chemical Engineering K-12 Outreach team took primary responsibility for teaching module development, and, along with AirU researchers and outreach staff, conducts outreach visits and installations at local high schools. The relationships with teachers developed over the years were invaluable in gathering teacher interest and trust that our outreach visits would be worthwhile to the teacher in meeting curricular goals.

This project has also partnered with a local community group, Breathe Utah (https://breatheutah.org/), which conducts public education and lobbying around the issue of air quality. Breathe Utah took responsibility for outreach visits to local middle schools, modifying the teaching module to better accommodate student sophistication and grade curriculum. Breath Utah was also instrumental in addressing district governmental hurdles.

Finally, teaching modules were developed in partnership with high school interns from the Academy for Math, Engineering, and Science (AMES). Our department maintains an internship program with AMES, a Title 1 high school, from which we gain about 20 interns each year. These interns worked on teaching module language and procedures, vetting that the concepts and activities would be effective in engaging our target cohort of citizens in this CS project [7].

Once appropriate teaching modules were developed (described below), they were used as a means to connect to teacher curriculum, engage classrooms, and train students, both on how their research sensor operates and how best to interpret AQ data. Chemical Engineering outreach 
students, AirU faculty, and Breathe Utah were all involved in conducting the designed teaching modules; AirU sensors are installed by the AirU faculty and sponsored staff. These partnerships between faculty, undergraduates, community partners, and local high schools were key to achieving CS goals. After installation, outreach teams and faculty remain available to troubleshoot and address student and teacher follow-up questions.

\section{Teaching Modules: Opening Up the Black Box}

An important part of collaborating with middle and high school students who are hoping to become citizen scientists is assuring they have the proper understanding of the science behind the research they will be conducting. An identified difficulty in the introduction of low-cost AQ sensors to the public is the citizens' perception of the sensor as a "black box," the workings of which are obscured [8]. Without proper understanding of the research tool the citizen will be using, engagement may suffer and students may easily reach erroneous conclusions about their data, possibly causing conflict with community partners. For instance, low-cost air quality sensors, like any sensor, may produce biased results, periodically drift, or report unreasonable particulate counts. However, many people who are not accustomed to the difficulties in obtaining and interpreting data tend to accept the numbers from their personal sensor with little skepticism, leading to either unnecessary alarm or complacency [9] . When their data conflicts with official government measures of air quality, some citizens have instead questioned the local Division of Air Quality and accused them of underreporting poor air quality, undermining partnerships essential to citizen science efforts.

To both aid CS participants in understanding their research sensor and its data and further facilitate connections with educators' learning objectives, we developed a hands-on design module in which students build an air quality sensor out of plastic building blocks (e.g. Legos ${ }^{\circledR}$ ) [7]. These student-built sensors mimic the low-cost, light-scattering research sensors, which they host as citizen scientists as part of the larger AQ\&U Project (Figure 2a, b, c). The intent of the module is to familiarize the students with how the sensor works and how it may fail to generate meaningful results. The activity is also used as a means to make additional curricular connections as discussed in the following section.

This module uses a white LED (IL051, Amazon) as the light source, a photoresistor (GL5516, Amazon) in series with a static $10 \mathrm{k} \Omega$ resistor in a voltage divider as a scattered light sensor, a mini $5 \mathrm{~V}$ brushless fan (Amazon) to pull air through the sensor, a neopixel strip as an indicator light (1426, Adafruit), and an arduino microcontroller to take readings and express data to the neopixel or to a laptop for plotting. Each of these parts has an analogous part on the Plantower research sensors which is a part of the AirU sensor package, helping students connect to their "black box" sensor in a hands-on manner. A detailed teaching module, parts list, Arduino code, and classroom presentation for this activity may be found on the AirU project's web site (https://airu.coe.utah.edu/) and is described in another published work [7].

Student teams design, build, and test light-scattering sensors within their class period (Figure 2d). During an orienting presentation, possible factors that may corrupt sensor data are discussed and related back to the AirU research sensor they will host. The module is delivered using a short presentation and a handout (both available on the AirU site:

https://airu.coe.utah.edu/) to explain the activity and the science behind it. 

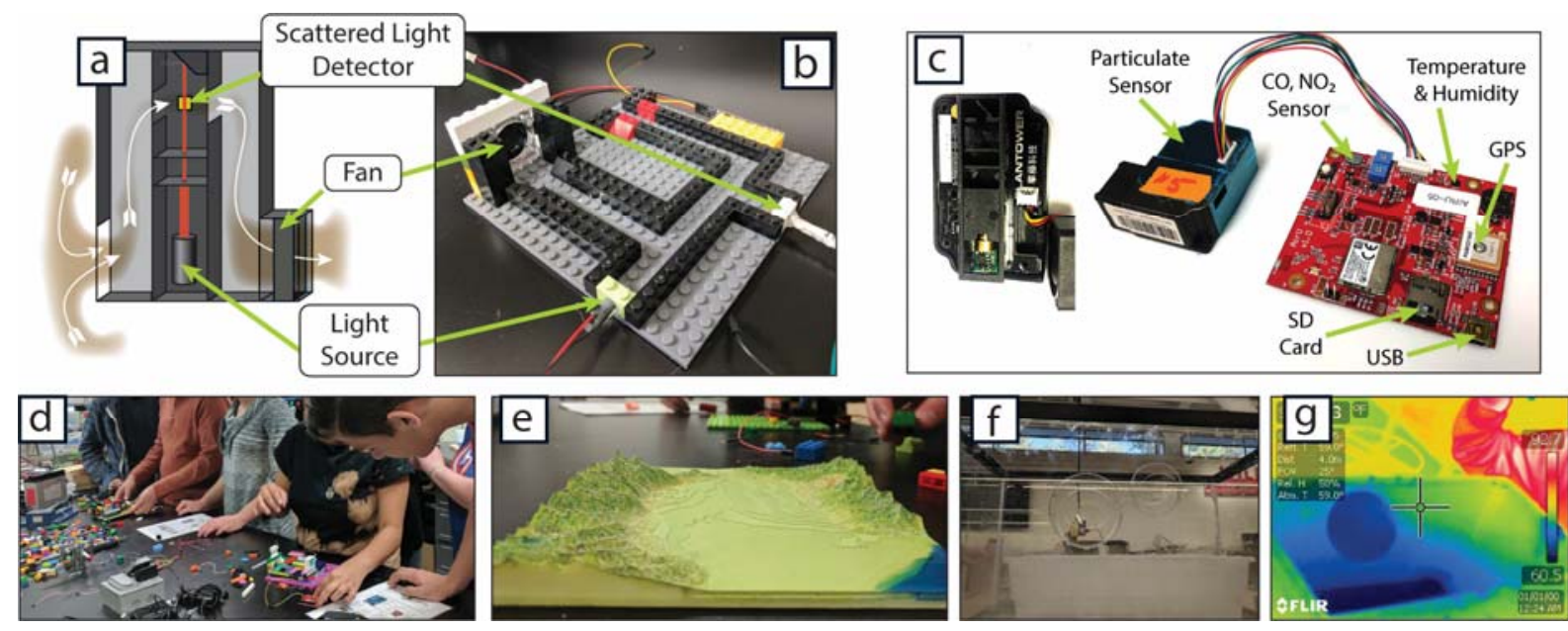

Figure 2: Outreach module Package. a) Illustration of sensor indicating how parts correlate with b) the building block sensor, which students build. c) Photograph of the sensor interior and AirU sensor package, used in "Care \& Feeding" module. d) Students assembling building block sensors in preparation to take possession of their research sensor. e) A 3D-printed model of the Salt Lake Valley, used to convey the science behind temperature inversions which lead to poor air quality. $\mathrm{f}$ ) Inversion demonstration in an aquarium, with bubbles floating on the inversion layer. e) Thermal image of a bubble floating on an inversion layer, used to discuss the how temperature inversions form.

Additional materials, such as a 3D-model of the student's valley (Figure 2e), are also used to better explain the science behind the causes of poor air quality. On the AirU site, STL files for 3D-printing of various valleys across the globe, which are known for their poor air quality, are made available.

This hands-on module is also augmented with a "Care and Feeding of Your AirU Sensor" activity, wherein students are given various real-world AQ data scenarios and asked to discuss possible reasons for their observations. Scenarios include: 1. Anomalous spikes in data, which could be caused by interruption of the light sources by an insect or by a passing smoker; 2 . Systematic error in the data, both proportional and through drift over time; 3. Legitimate spikes in data due to weather patterns and how to identify them; 4. Using sensors in close proximity to validate observations. By using such an activity, it is intended to insulate students somewhat from AQ data misinterpretation.

We also introduced an aquarium inversion module (Figure $2 \mathrm{f} \& 2 \mathrm{~g}$ ), to demonstrate how inversions form. In this module we create an inversion layer by placing dry ice in water at the bottom of a 10 gal aquarium. By blowing bubbles into the aquarium an inversion can be demonstrated; the bubble will seem to hover, floating on top of the inversion layer. Furthermore, if a lit match is dropped into the aquarium, the smoke from it will rise no higher than the inversion layer, visibly trapping the particulate matter. This teaching module is also posted on the AirU site.

Using these teaching modules, we are able to demonstrate: 1 . How our valley acts like a bowl; 2. How that bowl traps particulate pollution during temperature inversions; 3 . How we can detect that pollution; and 4 . How to judge the quality of particulate data. With this training we prepare students to take care of their research sensor and better understand its resulting data. 


\section{CS Hurdles: Creating Core Curricular Connections}

A significant barrier to entry for a college department attempting to interact with a public school classroom is often a lack of effective or apparent connection to the core curriculum of the grade and subject they wish to engage. In our experience, district administration may be resistive to an outreach visit until a clear curricular connection for the activity is described, even when local teachers are supportive. Private schools have greater flexibility in their curriculum and present an alternative when public schools are resistive. However, the demographics of private schools do not typically present opportunities to improve representation of first generation students and other underrepresented groups in engineering [10].

K-12 school faculty are generally required to meet a set of learning objectives, similar to those university faculty would detail in the objectives of a syllabus. All but eight US states have adopted common core state standards (http://www.corestandards.org/), but most curriculum standards for each particular jurisdiction are often detailed online and may be found with a simple internet search. Before a visit to a school is proposed, it is important to identify and assess how the team will specifically address the curricular needs of that particular classroom. For our particular project, we are targeting the core standards set by the state of Utah (https://www.uen.org/core/), but each state should have similar requirements.

Because this project intersects with topics from mathematics to environmental science and with six different grade levels, our approach has been to tailor the associated classroom activities to each visit. Instead of creating an individual teaching module for each course, we have created base modules and then connected them to the specific teacher's curriculum however they see fit, after consulting with them. For example, one engineering introduction class has used this activity to illustrate the use and function of voltage dividers. In response, we focused more on the circuity of their building block sensors during the two times we have visited this classroom. Another example would be at an environmental science classroom at a separate high school where we used the same AQ sensor activity (described above), but, instead, folded the material into the classroom's material on AQ health effects and the importance to communities of being able to detect pollutants; the circuitry for this classroom took a back seat. By tailoring the module to the instructor's curricular needs, we have been able to solicit as many classroom visits as could be managed by our outreach teams.

\section{CS Hurdles: School District Approval and Access to Wi-Fi Infrastructure}

In partnering with schools, a CS project may face a wide range of requirements from one school to another just down the street, depending on the district. Some districts have been very lax and hands-off, only requiring teacher approval, while others have required involvement of district managers and local politicians to allow student participation.

Furthermore, most all modern CS projects rely on internet access, which poses a difficulty when the project hopes to coordinate with K-12 public schools. Many schools understandably restrict student access to the internet and may be very hesitant to allow network access to any device, such as our AQ sensors, that poses a non-negligible security risk. As such, 
approximately half of the school districts we have interacted with have required a stringent vetting process with their IT managers. To obtain access, internet-capable CS devices must, at minimum, keep school Wi-Fi passwords encrypted.

Additionally, some districts have required memorandums of understanding to assure the school is not held legally responsible for damage to or loss of the CS research equipment, and that interactions with minors are properly supervised. Such agreements may introduce significant delays (two to three months) as legal departments become involved. Such delays should be anticipated for in timelines for CS projects that partner with K-12 schools.

\section{Results \& Discussion}

\section{CS K-12 Students Reached}

The developed model has been successful in reaching a substantial number of students over one year of its implementation (over 4,000). Figure 3 shows the number of cumulative students reached by this project over one project year. This plot includes only students in classrooms who were actively engaged with this CS project through related teaching modules, taking at least one full class period. The numbers of students engaged through this project at community functions and other tabling events is significantly higher, though with far less depth of interaction. Large jumps in the plot are typically due to visiting multiple classrooms on a single day (outreach visits often must take advantage of windows of undergraduate availability, and combined visits increase efficiency). Significant time spans between datum are primarily due to school holiday schedules. We have had no difficulty engaging as many high school teachers as we have resources to visit. The most significant bottlenecks on this CS project have been 1. obtaining district buy-in and 2. scheduling enough university staff to initiate CS class participation.

In addition to the 1,000 high school students and classrooms reached by our chemical engineering department, our community partner, Breathe Utah, has engaged over 3,000 middle school students with the same AirU CS teaching modules after being tailored to middle school curricular demands.

It should be noted that not all classrooms visited have received a sensor. Some have only been through the training step of the described CS model and will soon obtain a sensor. Some classes are still awaiting district approval, and a small minority were found to have no appropriate

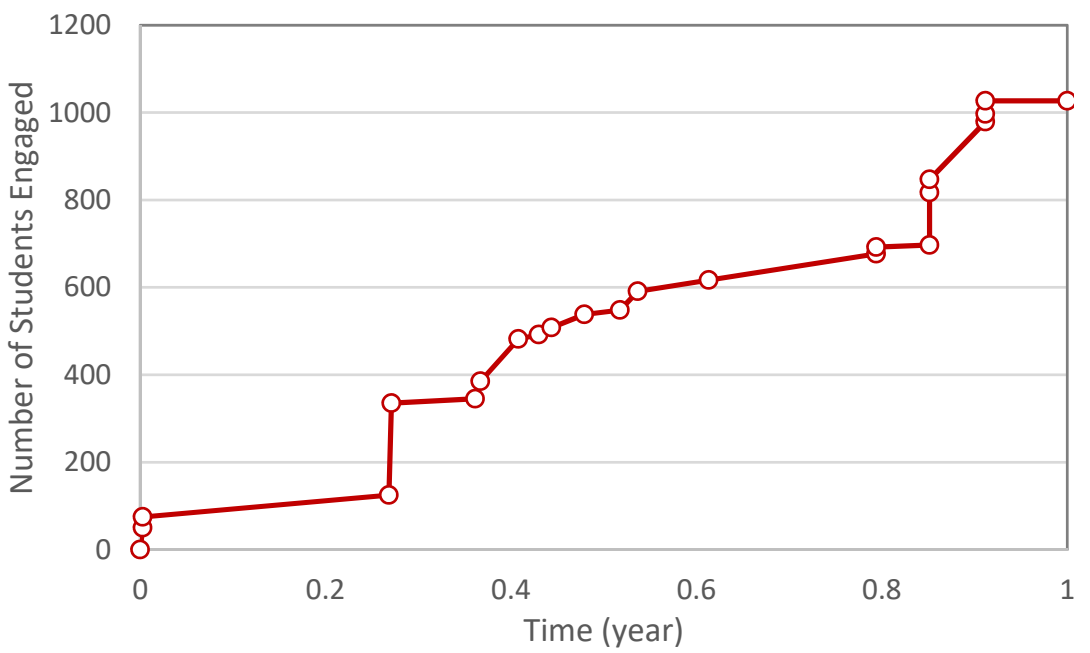

Figure 3: AQ CS Project Reach in Local Public Schools. The above plot shows one full project year of cumulative classroom visits for only high schools. Data for middle schools were not collected. 
location for a sensor (i.e., they lack shelter from weather and/or access to power or Wi-Fi). Currently, approximately 20 sensors have been placed through the K-12 outreach portion of this project ( 80 sensors in total have been placed throughout the valley). Of those adopted by schools, about half of them went to the homes of teachers or students in order to fill in geographical holes in the resulting AQ map.

Teachers and students asked several common questions during the school visits and after receiving the sensors. In particular, they wanted to understand what the sensor measures, what the numbers mean, how to compare the sensor readings to levels of health concerns, and what type of time averaging is relevant. The PMS sensor in the AirU sensor tends to track PM levels well, although they overestimate them [11], and we have been actively developing appropriate correction factors for the AirU's PM concentrations. CS questions about time averaging of air quality data are particularly insightful. The EPA and WHO set their air quality standards based on 24-hour averages, and guidance on the health effects of shorter time exposures has not been developed. Generally, interest in the sensors and the resulting data peaked in January and February of this year, months when our region typically experiences poor air quality. We anticipate interest to increase again beginning in November, as our inversion cycle repeats.

\section{CS Student \& Classroom Response}

We are limited in our collection of CS participant demographic and survey data due to the fact that most of our citizen scientists are minors. To collect research data on them, we would be required by our internal review board to obtain written parental consent for each student prior to our interaction with the students. As such, growth of this CS project necessarily precluded direct collection of data on participants. However, several important indications about our CS participants have been obtained.

Some survey data from a summer camp for high school girls were collected with approval (detailed in [7] and summarized here). While these campers could not take a sensor with them, they did participate in the building block module used to teach students how the CS AirU sensors function. The activity was assessed as "Great" by $65 \%$ and as "Good" by the remaining 35\%. Zero negative free form comments were collected from the 25 campers. Comments generally focused on how the activity caused the campers to more likely consider chemical engineering for their profession.

Free-form responses were also collected from a biotechnology course and an ELL (English Language Learner) science course (comments were translated from Spanish). Approximately $90 \%$ of the comments could be categorized as positive, primarily focusing on their appreciation for 1. gaining new knowledge about air quality, 2. learning through a hands-on activity, 3. connecting to a community health concern, and 4 . interacting with the AirU outreach team. All negative comments $(10 \%)$ focused on confusion on how to proceed with the activities, which may be expected with an open-ended hands-on project.

Of the classrooms engaged by this CS project in the 2016-2017 academic year, over $80 \%$ of them have invited our team to return to conduct additional teaching activities and sensor 
installs in the 2017-2018 academic year. Currently, the project is supporting five known student science fair projects (though, with over 1,000 students engaged, we have not been able to fully track such use of the resulting AQ map and are only aware of projects which request additional sensors or help). Additionally, this project has been highlighted on local and national press for engaging students on a STEM topic of great importance to the surrounding community [12], [13]. Lastly, one high school teacher who became involved with this CS project early on has been given an NSF Research Experience for Teachers award to help interpret the AQ data gained from the CS participants and further develop teaching material. By such measures, this project seems to have been successful in reaching and engaging middle and high school students as CS participants.

\section{Sensor and Network Performance}

We have found CS participants do require help maintaining (and being reminded to maintain) their sensors. Every morning, we run network diagnostics to identify sensors that have gone offline, and an undergraduate student contacts the sensor host to determine if the sensor is malfunctioning, if the Wi-Fi connection is problematic, or if a visit is necessary. Typically, we identify sensors going offline before the CS host does. Periodic problems with sensors include a weak Wi-Fi signal, loss of power, and PM sensor malfunction. The majority of the problems can be remedied by restarting the sensor, but approximately 4 sensors had to be repaired over one project year by either replacing the malfunctioning PM sensor or by reloading the firmware.

Some of the sensor hosts noticed problems with their sensor's measurements and contacted us. Figure 4 compares AirU

$\mathrm{PM}_{2.5}$ measurements to those from the nearest state monitoring station, 6.7 miles away. It illustrates that the AirU sensor generally tracks $\mathrm{PM}_{2.5}$ concentrations measured by the state, although it overestimates $\mathrm{PM}_{2.5}$ concentrations as previously reported [11]. It also illustrates two instances when the host contacted us because the PM readings exceeded expected ranges. In Figure 4(a), the Salt Lake Valley experienced a pollution episode. As PM levels declined in the valley, the AirU sensor spiked to 450 $\mu \mathrm{g} / \mathrm{m}^{3}$, and $\mathrm{PM}_{2.5}$ concentrations never

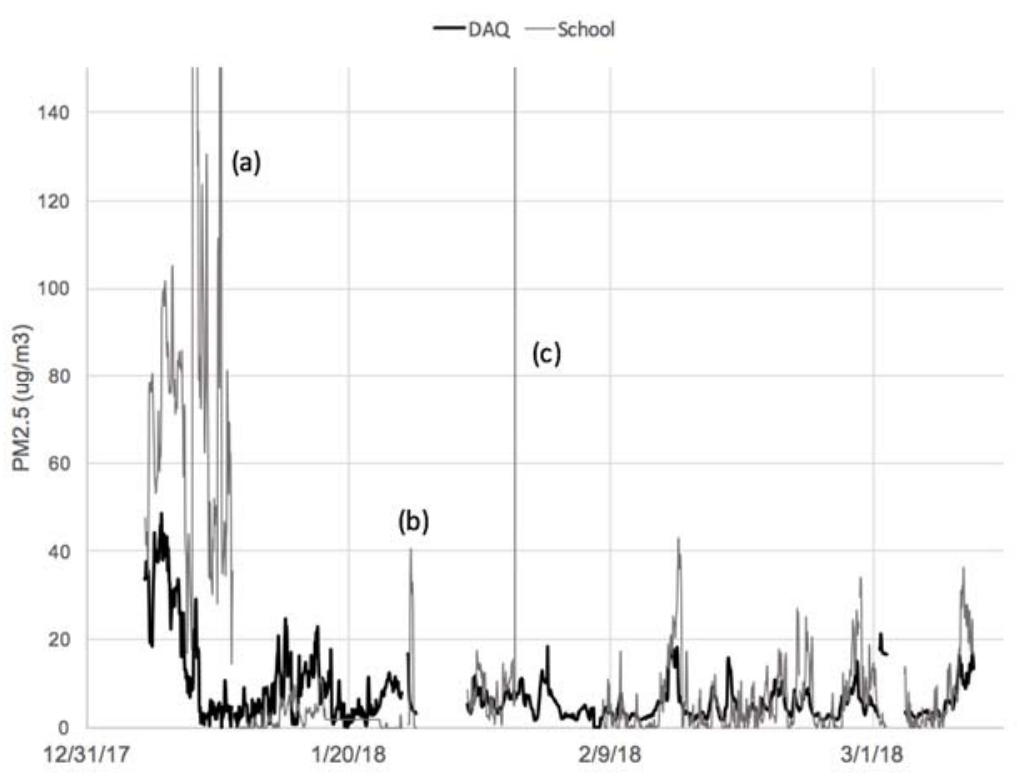

Figure 4: Hourly average $\mathrm{PM}_{2.5}$ concentrations measured by an AirU sensor and by a tapered element oscillating microbalance at a state monitoring station (DAQ). (a) AirU PM 2.5 readings spiked to $450 \mu \mathrm{g} / \mathrm{m}^{3}$ and did not return to background. (b) AirU sensor went offline and was restarted. (c) $\mathrm{PM}_{2.5}$ levels spiked to $5000 \mu \mathrm{g} / \mathrm{m}^{3}$ and remained at this level for one day. 
returned to reasonable clean air levels (below $15 \mu \mathrm{g} / \mathrm{m}^{3}$ ). In Figure 4(c), AirU PM2.5 levels reached the sensor's maximum reading of $5000 \mu \mathrm{g} / \mathrm{m}^{3}$ and remained at this level for a full day. The host reported no unusual activity in either case. In both cases, we replaced the AirU's PM sensor, and the sensor began tracking valley-wide PM levels. PM levels would not be expected to perfectly track PM levels 7 miles away, and periodic spikes could be due to an individual smoking near a sensor or some other activity. However, $\mathrm{PM}_{2.5}$ concentrations of $5000 \mu \mathrm{g} / \mathrm{m}^{3}$ for a full day are highly unlikely. This is more than 100 times the 24-hour national ambient air quality standard for $\mathrm{PM}_{2.5}$ of $35 \mu \mathrm{g} / \mathrm{m}^{3}$. It appears that when the AirU's PM sensor reaches some critical level, it may have difficulty returning to a baseline, and we are currently investigating whether this is a software or hardware problem. This type of problem was brought to our attention by our CS research and illustrates the type of observations from CS participants that may lead to greater understanding.

\section{Underrepresented Engagement}

One key goal of this CS project is to engage populations which are underrepresented in STEM fields, and this work would be expected to be particularly effective at such a task. Engineering projects that highlight the "helping profession" aspects of engineering have been associated with increased engagement of underrepresented populations [14], [15]. Furthermore, the CS teaching module activities in this work were developed in partnership with the student populations we most hoped to engage. A diverse team of five young aspiring women STEM professionals from the Academy for Mathematic, Engineering, and Science, a Title 1 high school in the Salt Lake valley, developed the building block teaching module.

While we cannot collect demographic data on the minors involved in this work, we do see evidence that we have been successful in reaching a more diverse population. According to public demographic data for the schools engaged by this project [16], this population has roughly double the percentage of students who are in underrepresented ethnic categories for our department (non-white, non-Asian), when compared to the surrounding community [17]. This work was also invited to be highlighted as a tabling event at the Northern Arapaho Environmental and Natural Resource Conference, which engaged native populations. In addition, this project was presented at the West Side Communities Breathe Clean Air Festival, and the Environment and You, West Side Festival, facilitated by Spanish-speaking outreach students. Finally, this work was highlighted at the Salt Lake Pride Festival. The direct and apparent impact of poor air quality on all communities and the potential of the research to affect community policy may make the associated outreach and CS efforts particularly amenable to reaching underrepresented populations.

\section{Translation into CS-Led Projects}

Lastly, we have seen evidence that citizen scientists and STEM students have begun using the AirU sensors and data in their own projects. To date, we are aware of five high-school student projects that rely on the AirU infrastructure. Two of these projects are from a Title I school. The projects range from correlating geographic differences in PM levels with pulmonary function to understanding how the distance from various pollution sources affects PM levels. In addition, the project has inspired chemical engineering students to deploy the AirU sensor on a drone, mechanical engineering students to develop an improved sensor housing, and a data 
informatics class to develop predictors of poor air quality. Students have also begun to take this project into new directions to improve the networks' usefulness, year-round. While particulate matter is the primary health concern in the winter months, ozone is the greatest air-quality concern during the summer months. A group of students have shown convincing preliminary data that the sensor's existing $\mathrm{NO}_{2}$ and $\mathrm{CO}$ sensor may be used to also estimate ozone concentration, potentially expanding the future impact of this project.

\section{Conclusions}

Using a combination of proven chemical engineering outreach methods, tailored teaching modules, and low-cost AQ networked sensors, we have been able to develop an active citizen scientist project within a chemical engineering department. Key to this CS project's reach has been to partner university faculty with local schools and community partners. Once significant hurdles were overcome, including school districts' legal and curricular connection concerns, this project's growth has only been limited by our ability to organize visits and provide enough sensors. While the air quality data from this project will take years to collect and analyze, a healthy and active network of CS participants has been established, and their data is available online and is now being used by university researchers and K-12 students alike to attempt to address an important community health concern.

\section{Acknowledgments}

This material is based in part upon work supported by the National Science Foundation under Grant Numbers NSF 1646408 and 1642513. Any opinions, findings, and conclusions or recommendations expressed in this material are those of the author(s) and do not necessarily reflect the views of the National Science Foundation.

We would like to thank the students of the Academy for Math, Engineering \& Science and West High School, who helped develop and hone this teaching module: Colin Pollard, Keenan Lins, Katie Nolan, Piper Stevens, Vaishnathi Thiraviyarajah, Annika Young, and Emma Dean. 


\section{References}

[1] E. Hand, "People Power," Nature, vol. 466, no. August, pp. 685-687, 2010.

[2] R. Simpson, K. R. Page, and D. De Roure, "Zooniverse: observing the world's largest citizen science platform,” Proc. 23rd Int. Conf. World Wide Web, pp. 1049-1054, 2014.

[3] S. Cooper et al., "Predicting protein structures with a multiplayer online game," Nature, vol. 466, no. 7307, pp. 756-760, 2010.

[4] Environmental Protection Agency, "Environmental Protection Belongs to the Public A Vision for Citizen Science at EPA National Advisory Council for Environmental Policy," 2016.

[5] C. Kullenberg and D. Kasperowski, "What is citizen science? - A scientometric metaanalysis," PLoS One, vol. 11, no. 1, pp. 1-16, 2016.

[6] C. Young and A. Butterfield, "Effective Engineering Outreach through an Undergraduate Mentoring Team and Module Database," Chem. Eng. Educ., vol. 48, no. 1, pp. 31-36, 2014.

[7] K. Le, A. Butterfield, K. Kelly, P.-E. Gaillardon, K. Tingey, and T. Becnel, "Building Air Quality Sensors \& Inspiring Citizen Scientists," Chem. Eng. Educ., vol. in press, 2018.

[8] Secure Workshop, "Use of low-cost sensor technology to monitor air quality \& engage citizens," no. March, 2016.

[9] M. Isaac, "Regulatory considerations of Lower Cost Air Pollution Sensor Data Performance," Environ. Manage., vol. 7, no. 4, pp. 32-37, 2014.

[10] J. T. Reardon, Sean F.|Yun, Private School Racial Enrollments and Segregation. Civil Rights Project, Harvard University, 124 Mt. Auburn Street, Suite 400 South, Cambridge, MA 02138. Tel: 617-496-6367; Fax: 617- 495-5210; e-mail: crp@harvard.edu; Web site: http://www.law.harvard.edu/civilrights/., 2002.

[11] K. E. Kelly et al., "Ambient and laboratory evaluation of a low-cost particulate matter," Environ. Pollut., vol. 221, pp. 491-500, 2017.

[12] "East High students create toy-block sensors to detect pollution | KSL.com." [Online]. Available: https://www.ksl.com/index.php?sid=43116735\&nid=148\&title=east-highstudents-create-toy-block-sensors-to-detect-pollution. [Accessed: 04-Feb-2018].

[13] "Kids Get Hands-on Education in Air Quality | Utah News | US News." [Online]. Available: https://www.usnews.com/news/utah/articles/2017-02-12/students-geteducation-in-air-quality-by-making-monitors. [Accessed: 04-Feb-2018].

[14] A. H. Inman, M. R. Anderson-rowland, M. Castro, and D. Zerby, "Assessing the Evolution of Engineering Saturday Academies for Middle School and High School 
Women," Women Eng. ProActive Netw., no. 480, 2003.

[15] L. Barrington and J. Duffy, "AC 2007-2871: ATTRACTING UNDERREPRESENTED GROUPS TO ENGINEERING WITH SERVICE-LEARNING Attracting Underrepresented Groups to Engineering with Service-Learning," ASEE Annu. Conf. Expo. Conf. Proc., 2007.

[16] S. L. C. S. District, "Fall Ethnicity," 2016. [Online]. Available: https://www.slcschools.org/schools/district-demographics/documents/document/2016-17ethnicity-report/english/2016-17-ethnicity-report-english.pdf. [Accessed: 02-Feb-2018].

[17] "United States Census Bureau. Quick Facts: Salt Lake County, Utah; Utah. United States Census.," 2016. [Online]. Available:

https://www.census.gov/quickfacts/fact/table/saltlakecountyutah,UT/PST045216. [Accessed: 12-Jan-2018]. 